\title{
Kyphoscoliotic Ehlers-Danlos syndrome due to lysyl hydroxylase 1 deficiency
}

INSERM

\section{Source}

INSERM. (1999). Orphanet: an online rare disease and orphan drug data base. Kyphoscoliotic Ehlers-Danlos syndrome due to lysyl hydroxylase 1 deficiency. ORPHA:1900

Ehlers-Danlos syndrome, kyphoscoliotic type (EDKT) is a form of Ehlers-Danlos syndrome characterized by severe hypotonia and kyphoscoliosis at birth, generalized joint hyperextensibility and ocular globe fragility. 